\title{
Multidisciplinary team approach for the management of patients with locally advanced non-small cell lung cancer: searching the evidence to guide the decision
}

\author{
In-Jae Oh, MD, PhD'1, Sung-Ja Ahn, MD, PhD² \\ Departments of ${ }^{1}$ Internal Medicine and ${ }^{2}$ Radiation Oncology, Chonnam National University Hwasun Hospital, Hwasun, Korea
}

Locally advanced non-small cell lung cancer (LA-NSCLC) is composed of heterogeneous subgroups that require a multidisciplinary team approach in order to ensure optimal therapy for each patient. Since 2010, the National Comprehensive Cancer Network has recommended chemoradiation therapy (CRT) for bulky mediastinal disease and surgical combination for those patients with single-station N2 involvement who respond to neoadjuvant therapy. According to lung cancer tumor boards, thoracic surgeons make a decision on the resectability of the tumor, if it is determined to be unresectable, concurrent CRT (CCRT) is considered the next choice. However, the survival benefit of CCRT over sequential CRT or radiotherapy alone carries the risk of additional toxicity. Considering severe adverse events that may lead to death, fit patients who are able to tolerate CCRT must be identified by multidisciplinary tumor board. Decelerated approaches, such as sequential CRT or high-dose radiation alone may be a valuable alternative for patients who are not eligible for CCRT. As a new treatment strategy, investigators are interested in the application of the innovative radiation techniques, trimodality therapy combining surgery after high-dose definitive CCRT, and the combination of radiation with targeted or immunotherapy agents. The updated results and on-going studies are thoroughly reviewed in this article.

Keywords: Patient care team, Combined modality therapy, Chemoradiotherapy, Radiation, Non-small-cell lung carcinoma

\section{Introduction}

Based on the evidence of a meta-analysis showing the superiority of concurrent chemoradiation therapy (CCRT) to sequential chemoradiation therapy (CRT) for unresectable stage III non-small cell lung cancer (NSCLC), with a 2- and 5 -year absolute survival benefits of $10 \%$ and $4.5 \%$, respectively [1], radiation oncologists must evaluate whether the patients are suitable for CCRT. Until now, the eligibility criteria for CCRT were not precisely defined except for the notion that CCRT is generally administered to patients with an Eastern Cooperative Oncology Group (ECOG) performance score (PS) of 0 or 1 , weight loss less than $5 \%-10 \%$ in the 3 months preceding the diagnosis, minimal or no comorbidities, and who are relatively young. Despite these formal selection criteria, a significant proportion of patients receiving CCRT experience poor compliance and severe toxicities leading to treatment interruption and even death. Tailored therapy requires more knowledge on the prognostic and predictive factors associated with survival or toxicity in patients undergoing CCRT. Primary tumor volume, the number of positive mediastinal lymph node stations, pretreatment hemoglobin $(\geq 12 \mathrm{~g} / \mathrm{dL})$, and forced expiratory volume in 1 second $\left(\mathrm{FEV}_{11}>2 \mathrm{~L}\right)$ are frequently reported as prognostic factors $[2,3]$. The tumor also should

Received 8 March 2017, Revised 21 March 2017, Accepted 22 March 2017.

Correspondence: Sung-Ja Ahn, MD, PhD, Department of Radiation Oncology, Chonnam National University Hwasun Hospital, 322 Seoyang-ro, Hwasun 58128, Korea. Tel: +82-61-379-7223, Fax: +82-61-379-7249, E-mail: ahnsja@chonnam.ac.kr

(C) This is an Open Access article distributed under the terms of the Creative Commons Attribution Non-Commercial License (http://creativecommons.org/ licenses/by-nc/4.0/) which permits unrestricted non-commercial use, distribution, and reproduction in any medium, provided the original work is properly cited.

www.e-roj.org 
be encompassed by a tolerable radiation therapy $(\mathrm{RT})$ target volume.

Another complicated question nowadays is the definition of the patient age range that is appropriate for CCRT. The increasing population of elderly lung cancer patients resulting from prolonged lifespans presents considerable challenges for the planning and delivery of cancer care services worldwide [4]. Current treatment decisions are often based on clinical judgement, which varies between clinicians and may also be subject to bias. However, the increased likelihood of using less aggressive forms of therapy out of fear of increased toxicity in elderly patients may contribute to the poor survival outcome in this population. More clinical data on the predictive factors for the compliance or toxicity in elder patients undergoing CCRT are necessary in order to avoid reversing the survival benefit of therapy. The identification of frailty is regarded as the best clinical practice standard and must be adopted in routine care [5]. Detection of the fittest elderly is important because functional rather than chronological age provides a broader view of possible treatment tolerance and survival [4].

The reports that the escalation of the radiation dose to 74 Gy do not further benefit patients with unresectable stage III NSCLC are challenging and require new disciplinary approaches. To date, there is no clear evidence that concurrent chemotherapy and hyperfractionated RT is superior to conventional CRT in terms of local control and survival $[6,7]$. One possible reason for the poorer survival in patients undergoing higher-dose radiation or hyperfractionated RT may be cardiopulmonary or esophageal toxicities [8]. In order to reduce these toxicities, innovative radiation techniques such as intensity-modulated radiotherapy (IMRT), image-guided radiotherapy (IGRT), adaptive $R T$, and proton beam therapy need to be developed. Furthermore, we need to consider surgical extirpation in patients with residual disease after standard CCRT using 60 Gy if further dose escalation does not provide a survival advantage. Induction or consolidation chemotherapy or maintenance therapy [9-11] failed to show a survival gain in combination with CCRT. Thus, the proven benefit of immunotherapy in stage IV lung cancer warrants its evaluation for use in earlier stages of lung cancer.

These challenging and complex multimodality treatment plans for the management of locally advanced NSCLC (LANSCLC) patients require the close coordination of caregiving professionals and should be performed at centers with experienced team whenever possible [12]. Therefore, the present study reviewed the published literature for informative evidences on the ideal complementation of the multidisciplinary team approach.

\section{Patient Fitness and Age Restrictions for CCRT}

In contrast to its survival benefit, CCRT also has a higher rate of grade 3 or 4 esophagitis than sequential CRT or RT alone; thus, CCRT compliance or tolerance should be estimated when selecting patients. The risk factors for the noncompliance include poor general PS, frailty, and comorbidities [13] (Table 1). However, undisputed predictors of survival other than PS

Table 1. Scored important comorbidity

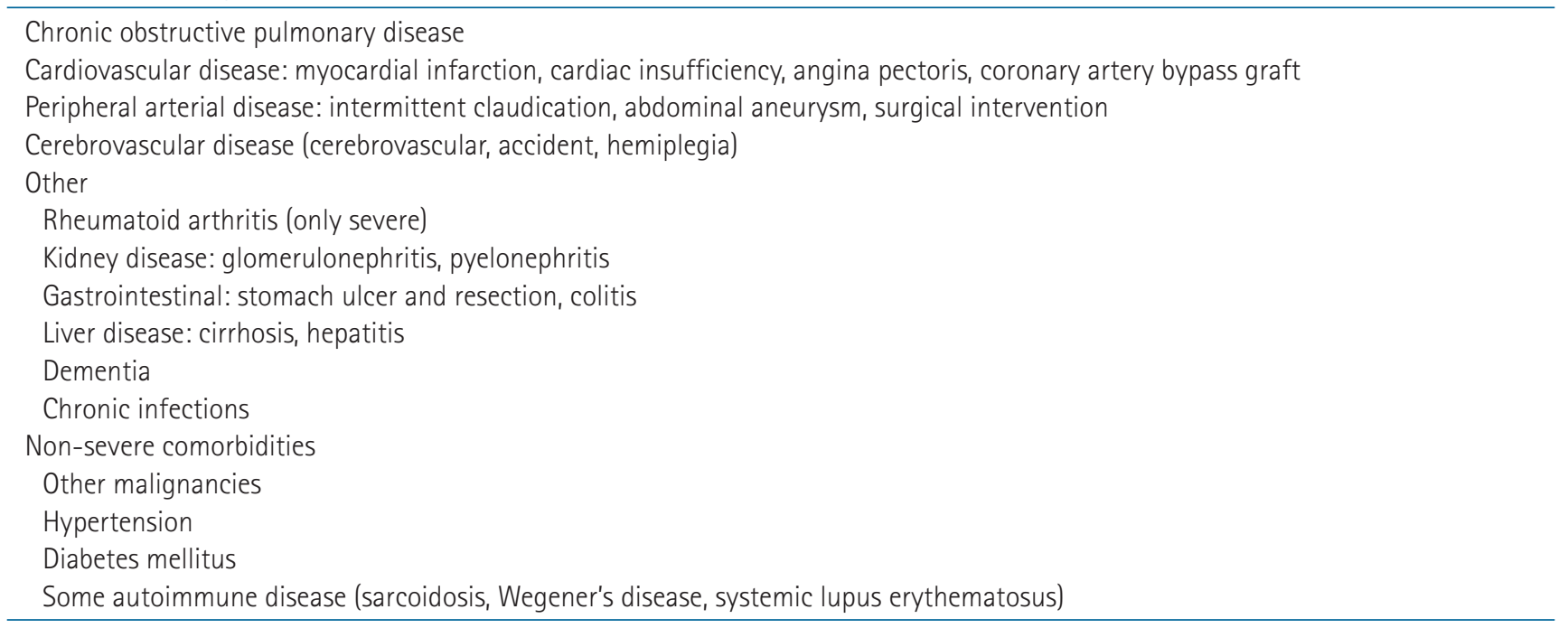

A slightly adapted version of the Charlson comorbidity index (CCI) [13]. 
Table 2. Criteria of adequate pulmonary function for definitive chest radiotherapy in lung cancer patients

\begin{tabular}{lc}
\hline $\mathrm{FEV}_{1}$ & $0.8 \mathrm{~L}$ \\
$\mathrm{FEV}$ & $40 \%$ \\
$\mathrm{FVC}$ & $45 \%$ \\
$\mathrm{DL}_{\mathrm{CO}}$ & $45 \%$ \\
$\mathrm{PaO}_{2}$ & $49 \mathrm{mmHg}$ \\
\hline
\end{tabular}

$\mathrm{FEV}_{1}$, forced expiratory volume in 1 second; $\mathrm{FVC}$, forced vital capacity; $\mathrm{DL}_{\text {cor }}$ diffusion lung capacity for carbon monoxide; $\mathrm{PaO}_{2}$, partial pressure of oxygen in arterial blood.

and weight loss that can be used in selecting patients for CCRT have not been identified. Accordingly, identification of clinically informative predictive factors and their relationship to CCRTinduced toxicity is valuable for the refinement of treatment strategies in patients with LA-NSCLC. A retrospective study showed that age $>75$ years $(p=0.009)$, diffusion lung capacity for carbon monoxide $\left(D_{c 0}\right) \leq 80 \%(p=0.011)$, and gross tumor volume (GTV) $\geq 100 \mathrm{~cm}^{3}(p=0.001)$ were statistically significant factors. Severe esophageal and lung toxicity and interruption of RT were more frequent in patients with multiple adverse prognostic factors [14].

Chronological age alone is a poor predictor of cancer treatment tolerance [15]. The inclusion parameters in the representative clinical trials were age (maximum 74 years); ECOG PS (a maximum of 2); weight loss $<10 \%$ in the last 3 months; tolerable lung functions (Table 2), and adequate cardiac, renal and hematological functions.

More than half of the patients with stage III are considered ineligible for concurrent regimens if inclusion is restricted to patients less than 75 years of age and those with less than two serious comorbidities [13]. However, with the rising numbers of patients older than 75 years without significant comorbidities in clinical practice and the lack of knowledge on treatment outcomes in elderly patients, the exclusion of patients for CCRT due to age should be re-evaluated. There remain controversial results regarding age factors (Table 3 ). Several studies have reported that CCRT could benefit appropriately selected elderly patients $[14,16]$. In a subgroup analysis of elderly patients ( $\geq 75$ years), the median survival time was 31 months for those with adequate pulmonary function $\left(\mathrm{DL}_{\mathrm{co}}>80 \%\right)$, with a small tumor volume $\left(\mathrm{GTV}<100 \mathrm{~cm}^{3}\right)$. Although the number of elderly patients $(n=11)$ was too small to verify the statistical significance of these findings, it appears that elderly patients ( $\geq 75$ years) with no adverse risk factors may benefit from CCRT [14]. Heterogeneity of the older cancer patient population requires a carefully tailored approach that considers individual frailty. The International Society of Geriatric Oncology has recently published updated guidance on the use of geriatric assessment in older cancer patients [17]. This review supports the routine assessment of individual frailty and fitness regardless of age to guide treatment decisions.

\section{Strategy Reducing the Radiation-Related Toxicities}

Improved RT treatment techniques that prevent severe adverse effects may enhance the survival outcomes [18]. Quality of life (0OL) analysis of the RTOG 0617 demonstrated a clinically meaningful decline in $\mathrm{OOL}$ in the 74-Gy arm at 3 months and identified a significantly lower decline in patientreported $\mathrm{OOL}$ after treatment with IMRT compared with that of 3D-CRT up to one year after completion of treatment. Meta-analysis of overall survival (OS) in 3,795 patients with NSCLC randomized in 21 trials to compare higher versus lower RT doses of curative intent showed that higher RT doses with chemotherapy led to poorer survival, but those without chemotherapy resulted in longer survival [19]. These findings support the consideration of modern treatment methods such as IMRT, IGRT, respiration-gated RT, and adaptive RT to reduce toxicity, especially in combination with chemotherapy.

The first step for the implementation of the high-quality radiation treatment planning is to delineate the real and correct target (Table 4). Yap et al. [20] performed a planning

Table 3. Relationship between age and survival

\begin{tabular}{|c|c|c|c|c|c|}
\hline Clinical trials & Phase & No. of patients & Age (yr) & $\operatorname{HR}(95 \% \mathrm{Cl})$ & $p$-value \\
\hline $\begin{array}{l}\text { CALGB, combined of } \\
8433,8831,9130,9431,9534\end{array}$ & III & 704 & $\geq 70$ & $1.07(0.88-1.31)$ & 0.5 \\
\hline RTOG 9410 & III & 577 & $\geq 60$ & - & 0.001 \\
\hline Hoosier Oncology Group & III & 203 & $\geq 70$ & $1.55(0.92-2.62)$ & 0.101 \\
\hline CALGB 39801 & III & 331 & $\geq 70$ & $1.45(1.11-1.90)$ & 0.01 \\
\hline Spanish Lung Cancer Group 0008 & $\|$ & 139 & $\geq 70$ & $1.14(0.67-1.96)$ & 0.621 \\
\hline
\end{tabular}

$\mathrm{HR}$, hazard ratio; $\mathrm{Cl}$, confidence interval. 
Table 4. Schema for defining mediastinal GTV in NSCLC

\begin{tabular}{|c|c|c|c|}
\hline Nodal diameter (short axis) & PET status & Approach & Comment \\
\hline$<1 \mathrm{~cm}$ & + & Include in GTV & $\begin{array}{l}\text { Positive predictive value higher for } \\
\text { PET than CT, biopsy when possible }\end{array}$ \\
\hline$<1 \mathrm{~cm}$ & - & Exclude from GTV & $\begin{array}{l}\text { High negative predictive value for PET, } \\
\text { small probability of N2 disease }\end{array}$ \\
\hline$>1 \mathrm{~cm}$ & + & $\begin{array}{l}\text { Include in GTV unless pathology is } \\
\text { negative }\end{array}$ & $\begin{array}{l}\text { Sensitivity for TBNA is inferior to EBUS or } \\
\text { EUS-TBNA }\end{array}$ \\
\hline 1-1.5 cm (no cytology available) & - & $\begin{array}{l}\text { Exclude from GTV if primary tumor is } \\
\text { PET-positive, unless cytology or } \\
\text { histology is positive }\end{array}$ & $\begin{array}{l}\text { High negative predictive value for } \mathrm{PET}, \\
\text { small probable (5\%) of N2 disease }\end{array}$ \\
\hline$>1.5 \mathrm{~cm}$ & - & Include in GTV & $21 \%$ probability of $\mathrm{N} 2$ disease \\
\hline
\end{tabular}

GTV, gross tumor volume; NSCLC, non-small cell lung cancer; PET, positron emission tomography; CT, computed tomography; TBNA, transbronchial needle aspiration; EBUS, endobronchial ultrasound; EUS, endoscopic ultrasound.

study based on serial respiratory-gated ${ }^{18} \mathrm{~F}$-fluorodeoxyglucose (FDG) PET/CT scans during RT to investigate the dosimetric benefits of a PET-based adaptive dose-escalation technique at weeks 0,2 , or 4 during RT concurrent with chemotherapy. The median doses received by $95 \%$ of the planning target volume $\left(D_{95}\right)$ at weeks 0,2 , and 4 to the FDG-avid primary tumor and PET-nodal disease were 74.4, 75.3, and 74.1 Gy and 74.3, 71.0, and $69.5 \mathrm{~Gy}$, respectively. The authors concluded that the use of ${ }^{18} \mathrm{~F}-\mathrm{FDG}-4 \mathrm{DPET} / 4 \mathrm{DCT}$ allowed dose escalation of both primary and nodal disease in most patients.

Dosimetric parameters of $V_{51} V_{20}$ and mean lung dose (MLD) has been considered as the risk factors for radiation pneumonitis (RP) $[18,21]$. MLD and $V_{20}$ are the most commonly used lung dose constraints, with recommended MLD and $V_{20}$ limits of $<20-23$ Gy and $<30 \%-35 \%$, respectively, in clinical practice [22]. RTOG 0617 study showed that although the patients treated with IMRT had larger and more advanced tumors, IMRT was associated with less $\geq$ grade 3 pneumonitis (7.9\% vs. 3.5\%, $p=0.039$ ) and the lung volume receiving $\geq 5$ Gy $\left(V_{5}\right)$ was not associated with any $\geq$ grade 3 toxicity, whereas the lung $V_{20}$ was associated with increased $\geq$ grade 3 pneumonitis risk in multivariate analysis $(p=0.026)$ [23]. Authors argue that the findings, despite significantly greater lung $V_{5}$ values, IMRT was associated with a better lung toxicity profile than 3D-CRT do not support lung $V_{5}$ as a predictor of toxicity in the RT of LA-NSCLC [23]. The authors also argue against using lung $V_{5}$ for the optimization of IMRT plans because an attempt to lower the $V_{5}$ may lead to less conformity in the high-dose regions and an inability to reduce the intermediate dose $\left(\mathrm{V}_{20}\right)$.

Adding functional dose-volume parameters calculated from perfusion single-photon emission computed tomography
(SPECT) resulted in higher sensitivity and specificity for the detection of RP, the association between functional parameters and RP risk was independent of tumor size, disease stage, and total lung volume [24]. All patients with functional MLD over 16 Gy developed RP. Therefore, the constraint of F-MLD of $16 \mathrm{~Gy}$ may be useful in functional dose planning to predict the risk of RP. Several other techniques besides SPECT may facilitate the delineation of regional pulmonary function for curative RT of NSCLC, including hyperpolarized ${ }^{3} \mathrm{He}$ and ${ }^{129} \mathrm{Xe}$ magnetic resonance imaging and $4 \mathrm{D}-\mathrm{CT}[25,26]$.

Sparing the heart could be crucial to survival based on recent data from the RTOG 0617 study. Heart $V_{5}$ and $V_{30}$ are both important predictors of patient survival [8]. Data from Washington University and Barnes-Jewish Hospital have tracked cardiac events in patients treated for LA-NSCLC with CRT and show that keeping the heart $\mathrm{V}_{50}<25 \%$ is the best metric within this dataset [27]. To determine the impact of heart dose on early 2-year OS in the RTOG 0617 study, Tucker et al. [28] retrospectively reviewed heart and lung dose-volume histograms extracted from patients with stage III NSCLC with CCRT using 3D-CRT, IMRT, or proton therapy. The significant factors in multivariate analysis were PS, GTV, and MLD. No evidence was found that heart dose had an independent effect on OS during the first 2 years. Considering the variability in heart contouring, corrected results using re-contoured heart structures and longer follow-up may provide critical insights into the effects of radiation doses on specific anatomic regions of the heart.

Adaptive RT can be advantageous in allowing small volumes of residual tumor to be treated to greater doses without exceeding the tolerance of the surrounding normal tissues. The RTOG 1106 protocol is an ongoing randomized phase II 
study comparing 60 Gy given in 30 fractions with IMRT in the standard arm versus an experimental arm that uses a midtreatment PET-adapted hypofractionated RT boost to intensify the radiation dose to residual tumor volumes during a total duration of 30 fractions ranging from 60 to $84 \mathrm{~Gy}$ in the final nine fractions, limiting the mean lung dose $<20 \mathrm{~Gy}$.

The consensus statement on proton therapy indicates that because of its Bragg peak, proton RT is a promising modality for delivering adequate dose to the target to potentially improve local control and survival and decrease side effects in patients with NSCLC [29]. Long-term outcomes after proton therapy of 60-74.1 Gy (relative biological effectiveness [RBE]) with concurrent chemotherapy showed that the median survival time was 30.4 months and the 5 -year OS rates were $25.3 \%$ and $31.8 \%$ in stages IIIA and IIIB, respectively [30]. These results can be explained by the potential advantage of proton beam therapy to safely deliver a relatively high radiation dose of 74 Gy (RBE) while minimizing cardiopulmonary toxicity. The RTOG 1308 trial is a phase III trial of protons versus photons (IMRT or 3D-CRT) to $70 \mathrm{~Gy}$ (RBE) with concurrent chemotherapy while applying strict dose volume constraints to adjacent normal tissues for the treatment of patients with locally advanced, inoperable NSCLC (ClinicalTrials.gov ID: NCT02394548).

\section{Strategy Combining Surgery after Full-Dose CCRT}

Investigators continue to explore the concept of pulmonary resection after induction CRT in an effort to improve both local control and OS. In a retrospective study of preoperative CCRT (44-45 Gy) with etoposide/cisplatin (EP) or docetaxel/cisplatin in patients with N2-positive stage IIIA NSCLC ( $=355$ ), ypN stage was the most important prognostic factor for survival; neither initial bulk nor the extent of cN2 disease influenced the prognosis [31]. Patients with stage III NSCLC treated with surgery after induction CCRT and with pathologically complete lymph node response showed significantly higher survival compared to that of patients with CCRT only (81 vs. 31.8 months; $p=0.0004$ ) and patients with residual nodal disease after surgery experienced poor OS (median, 16.1 months) [32]. Although the preoperative radiation dose has traditionally been limited to $45 \mathrm{~Gy}$, primarily owing to concerns of excess postoperative morbidity and mortality, the hypothesis that full-dose of CRT would result in higher pathologic complete response and excellent long-term survival requires testing. No solid phase III data are available on the role of surgery after a radical CRT schedule and upfront patient selection criteria for this strategy remain unknown. The best candidates who can benefit from surgery are a specific patient subgroup with mediastinal nodal clearance who undergo a complete resection.

Surgery is associated with a potentially greater risk of complications, particularly stump breakdown and bronchopleural fistula, in a field that has received definitive doses of CCRT ( $\geq 60 \mathrm{~Gy}$ ). Thus, it was understood that any attempt to deliver doses higher than 45 Gy would have to be associated with strategies designed to mitigate the risk of postoperative pulmonary toxicities. Investigators at the University of Maryland documented the ability to safely perform pulmonary resections in the setting of full-dose CRT by incorporating the use of vascularized muscle flaps to cover bronchial stumps, careful limitation of intraoperative fluid administration, and avoidance of postoperative barotraumas [33]. When a pedicled muscle flap is employed to buttress the closed lobar bronchus, bronchopleural fistula was exceedingly rare and pulmonary resection was feasible and safe even after doses preoperative radiation of $60 \mathrm{~Gy}$ or higher [34]. Major morbidity occurred in 17\% of patients and the mortality rate was 2.3\%; there were no bronchopleural fistulas after lobectomy, but two occurred after right pneumonectomy. Patients undergoing pneumonectomy experienced an unacceptably high rate of postoperative mortality due to acute respiratory distress syndrome and other respiratory causes [35]. The role of surgery in stage III disease is limited to those patients with adequate down-staging after induction therapy with residual tumor and who do not require a pneumonectomy [36].

Patients treated with neoadjuvant therapy are allowed a period of 4 to 6 weeks for recovery before surgical resection. During this time, restaging is performed with a PET/CT to assess the response to the preoperative therapy. Surgery should be planned to be performed within 8 weeks of the completion of induction therapy in suitable patients. The normal tissue dose constraints for the successful accomplishment of trimodality therapy with high-dose RT should be more tightly controlled than that of CRT or RT alone (Table 5). In a retrospective study on dosimetric parameters and pulmonary functions, operative mortality was statistically significantly higher in patients with higher lung $V_{5}$ or $V_{10}$. The cut-off receiver operating characteristic (ROC) curve for lung V10 relative to the operative mortality was $27.5 \%$ (sensitivity, 83.3\%; specificity, 75\%). Lung MLD and $V_{20}$ were insignificant in this study [37]. 
Table 5. Normal tissue dose constraints for thorax irradiation in lung cancer patients

\begin{tabular}{lccc}
\hline \multicolumn{1}{c}{ Organ } & RT alone & CCRT & Trimodality \\
\hline Spinal cord (Gy) & 50 & 45 & 45 \\
Lung & & & \\
MLD (Gy) & $<20$ & $<20$ & $<20$ \\
$V_{20}(\%)$ & $<35$ & $<35$ & $<20$ \\
$V_{10}(\%)$ & $<45$ & $<45$ & $<40$ \\
$V_{5}(\%)$ & $<65$ & $<65$ & $<55$ \\
Liver & & & \\
$V_{30}(\%)$ & $<40$ & $<40$ & $<40$ \\
Esophagus & & & \\
$D_{\text {max }}(G y)$ & $<75$ & & \\
$V_{60}(\%)$ & $<50$ & $<50$ & \\
$V_{55}(\%)$ & & & \\
$V_{50}(\%)$ & & & \\
Heart & & & \\
$V_{40}(\%)$ & $<50$ & & \\
Kidney & & & \\
$V_{20}(\%)$ & $<50$ & & \\
\hline
\end{tabular}

$R T$, radiotherapy; CCRT, concurrent chemoradiotherapy; MLD, mean lung dose.

The RTOG-0229 study was designed to evaluate the impact of full-dose radiation on the ability to sterilize known mediastinal nodal disease. It is encouraging to note that this study has documented the ability to safely perform pulmonary resection after an induction regimen that includes full-dose radiation. The study results support the contention that fulldose radiation delivered to known mediastinal disease can improve nodal clearance rates [38]. The low incidence of postoperative mortality in this trial represents the impact of both the experience of the participating surgeons and the adherence to strategies designed to minimize postoperative morbidity and mortality. The results imply that, with appropriate surgical expertise and tighter normal tissue dose constraints, an aggressive multimodal approach to LA-NSCLC is possible. Based on the success of this study, a randomized phase II trial (RTOG-0839) was designed to evaluate the potential benefits associated with the addition of an epidermal growth factor receptor (EGFR) inhibitor, panitumumab, to this induction regimen.

\section{Strategy Combining the New Anticancer Agents}

Weekly paclitaxel and carboplatin has emerged as a welltolerated and efficacious CCRT regimen. The most recent phase III randomized trial evaluating the modern pemetrexed and cisplatin followed by pemetrexed consolidation regimen versus standard CRT with EP in patients with non-squamous histology was stopped early for futility after enrolling 555 patients [39]. OS for the pemetrexed and cisplatin arm was not superior to that in the PE arm. Thus, platinum-based doublet chemotherapy should be supported as concurrent regimens and weekly paclitaxel and carboplatin or cyclic EP remain the most commonly administered regimens [40].

After the RTOG-0324 phase II study, which identified an impressive median OS of 22.7 months with the addition of cetuximab [41], the RTOG-0617 study was modified to a 2 $\times 2$ factorial design in order to also evaluate the addition of cetuximab to CRT. The median survival in patients who received cetuximab was 25 months compared with 24 months in those who did not (hazard ratio [HR], 1.07; $p=0.29$ ) and the OS were similar with or without cetuximab [8]. The addition of erlotinib or gefitinib also did not result in superior outcomes. In particular, erlotinib administered following consolidation chemotherapy and concurrent CRT to a cohort of patients without EGFR mutations resulted in significantly decreased survival [42]. The RTOG 1306 is an ongoing randomized phase II trial of induction erlotinib or crizotinib for 12 weeks followed by standard treatment using weekly paclitaxel/carboplatin or cyclic EP with radiation versus standard treatment alone. Similarly, a randomized trial investigating the addition of thalidomide as an antiangiogenic compound showed no difference in survival. Other antiangiogenic factors such as bevacizumab have also been shown to be toxic or ineffective when added to CRT [43]. Other drugs that have been reevaluated for lung cancer therapy in the research setting include statins, $\beta$-blockers, itraconazole, and non-steroidal anti-inflammatory drugs (NSAIDs) [44].

Strategies for combining the use of ionizing radiation and immunomodulators have been proposed. A randomized phase III trial of therapeutic cancer vaccine strategies after CRT in stage III disease has been completed. The Stimulating Targeted Antigenic Response to NSCLC (START) trial evaluated tecemotide, an MUC1 antigen-specific immunotherapy that induces a T-cell response to MUC1, a commonly overexpressed antigen on lung cancer cells [45]. The trial assessed an MUC1 vaccine in stage III NSCLC patients who had response or stable disease after standard CRT. CRT was delivered concurrently or sequentially. Patients were randomized to receive maintenance tecemotide after CRT. The modified intention-to-treat population included 1,239 patients. The primary endpoint was not met (adjusted $\mathrm{HR}, 0.88 ; 95 \%$ confidence interval, 0.75 $1.03 ; p=0.123)$. There are a number of possible explanations 
for these negative results. One primary reason is that cancer vaccines, when used alone, fail to address the many immunosuppressive factors in the tumor microenvironment.

PACIFIC trial (NCT 20125461) is a randomized phase III, double-blinded, international trial to evaluate the efficacy and safety of durvalumab, an anti-programmed death ligand 1 (anti-PDL1) antibody in patients with unresectable stage III NSCLC who have not progressed after definitive, platinumbased, CCRT. This trial completed accrual in April 2016 and has randomized more than 700 patients. In addition to the important efficacy outcomes, a number of exploratory objectives will assess tissue and blood for possible biomarkers. Ipilimumab, an antibody that targets cytotoxic T-lymphocyteassociated antigen (CTLA-4), is the first FDA-approved anticancer antibody that specifically targets non-malignant immune cells. The data presented indicated that, in tumors that are refractory to treatment with the CTLA-4 blockade alone, the combination with RT to one tumor site can induce systemic tumor control and in some cases, complete tumor regression [46]. The question of the doses and fractionations used for radiation becomes more relevant when designing new trials to incorporate immunotherapy agents, such as antiPD1 or anti-PDL1. Hypofractionated doses may enhance the inflammatory response, inducing greater recruitment of $T$ cells, and serve as a 'fire starter', rather than the fire itself, igniting the host's immune response [47]. One of the ongoing concerns regarding immune checkpoint blockage is the risk of initiating auto-immunity and inflammation, such as pneumonitis, that could be exacerbated by radiation. The combination treatment could increase the incidence and severity of pneumonitis in NSCLC patients [48].

Another interesting class of agents being examined is DNA repair inhibitors. SWOG is conducting a phase $1 / I I$ trial evaluating the addition of the poly-ADP-ribose polymerase inhibitor veliparib to CCRT as a potential chemosensitizer and radiosensitizer.

\section{Conclusion}

The objectives of the multidisciplinary approach for the management of LA-NSCLC are to achieve the most benefit from the regimen while minimizing side effects. The clinical team continuously reviews the patient's tolerance to the treatment regimen, encourages patients to report all side effects, and provides supportive treatment for the side effects when possible. CCRT may be changed to a less aggressive treatment such as sequential CRT and RT alone, or to accelerated regimens such as trimodality treatment in selected patients. Innovative radiation techniques should be employed reducing the toxicities. Optimal strategies for the combination of targeted or immunomodulating agents have not yet been determined.

\section{Conflict of Interest}

No potential conflict of interest relevant to this article was reported.

\section{Acknowledgments}

This study was supported by a grant from the National RetD program for Cancer Control, Ministry of Health \& Welfare of Korea (820010).

\section{References}

1. Auperin A, Le Pechoux C, Rolland E, et al. Meta-analysis of concomitant versus sequential radiochemotherapy in locally advanced non-small-cell lung cancer. J Clin Oncol 2010;28:2181-90.

2. Dehing-Oberije $C$, De Ruysscher $D$, van der Weide $H_{\text {, et al }}$ Tumor volume combined with number of positive lymph node stations is a more important prognostic factor than TNM stage for survival of non-small-cell lung cancer patients treated with (chemo)radiotherapy. Int J Radiat Oncol Biol Phys 2008;70:1039-44.

3. Ademuyiwa FO, Johnson CS, White AS, et al. Prognostic factors in stage III non-small-cell lung cancer. Clin Lung Cancer 2007;8:478-82.

4. Handforth C, Clegg A, Young C, et al. The prevalence and outcomes of frailty in older cancer patients: a systematic review. Ann Oncol 2015;26:1091-101.

5. Ellis G, Whitehead MA, Robinson D, O'Neill D, Langhorne P. Comprehensive geriatric assessment for older adults admitted to hospital: meta-analysis of randomised controlled trials. BMJ 2011;343:d6553.

6. Curran WJ Jr, Paulus R, Langer CJ, et al. Sequential vs. concurrent chemoradiation for stage III non-small cell lung cancer: randomized phase III trial RTOG 9410. J Natl Cancer Inst 2011;103:1452-60.

7. Baumann $M$, Herrmann $T$, Koch $R$, et al. Final results of the randomized phase III CHARTWEL-trial (ARO 97-1) comparing hyperfractionated-accelerated versus conventionally fractionated radiotherapy in non-small cell lung cancer (NSCLC). Radiother Oncol 2011;100:76-85.

8. Bradley JD, Paulus R, Komaki $R$, et al. Standard-dose versus 
high-dose conformal radiotherapy with concurrent and consolidation carboplatin plus paclitaxel with or without cetuximab for patients with stage IIIA or IIIB non-small-cell lung cancer (RTOG 0617): a randomised, two-by-two factorial phase 3 study. Lancet Oncol 2015;16:187-99.

9. Vokes EE, Herndon JE 2nd, Kelley MJ, et al. Induction chemotherapy followed by chemoradiotherapy compared with chemoradiotherapy alone for regionally advanced unresectable stage III Non-small-cell lung cancer: Cancer and Leukemia Group B. J Clin Oncol 2007;25:1698-704.

10. Hanna N, Neubauer M, Yiannoutsos C, et al. Phase III study of cisplatin, etoposide, and concurrent chest radiation with or without consolidation docetaxel in patients with inoperable stage III non-small-cell lung cancer: the Hoosier Oncology Group and U.S. Oncology. J Clin Oncol 2008;26:5755-60.

11. Ahn JS, Ahn YC, Kim JH, et al. Multinational randomized phase III trial with or without consolidation chemotherapy using docetaxel and cisplatin after concurrent chemoradiation in inoperable stage III non-small-cell lung cancer: KCSGLU05-04. J Clin Oncol 2015;33:2660-6.

12. Schild SE, Vokes EE. Pathways to improving combined modality therapy for stage III nonsmall-cell lung cancer. Ann Oncol 2016:27:590-9.

13. De Ruysscher $D$, Botterweck $A$, Dirx M, et al. Eligibility for concurrent chemotherapy and radiotherapy of locally advanced lung cancer patients: a prospective, populationbased study. Ann Oncol 2009;20:98-102.

14. Kim YH, Ahn SJ, Kim YC, et al. Predictive factors for survival and correlation to toxicity in advanced Stage III non-small cell lung cancer patients with concurrent chemoradiation. Jpn J Clin Oncol 2016;46:144-51.

15. Quoix E, Zalcman G, Oster JP, et al. Carboplatin and weekly paclitaxel doublet chemotherapy compared with monotherapy in elderly patients with advanced non-small-cell lung cancer: IFCT-0501 randomised, phase 3 trial. Lancet 2011;378:107988.

16. Takigawa N, Kiura K, Segawa $Y$, et al. Benefits and adverse events among elderly patients receiving concurrent chemoradiotherapy for locally advanced non-small cell lung cancer: analysis of the Okayama Lung Cancer Study Group trial 0007. J Thorac Oncol 2011;6:1087-91.

17. Wildiers $H_{1}$ Heeren $P$, Puts $M$, et al. International Society of Geriatric Oncology consensus on geriatric assessment in older patients with cancer. J Clin Oncol 2014;32:2595-603.

18. Movsas B, Hu C, Sloan J, et al. Quality of life analysis of a radiation dose-escalation study of patients with non-smallcell lung cancer: a secondary analysis of the Radiation Therapy Oncology Group 0617 randomized clinical trial. JAMA Oncol 2016:2:359-67.

19. Ramroth J, Cutter DJ, Darby SC, et al. Dose and fractionation in radiation therapy of curative intent for non-small cell lung cancer: meta-analysis of randomized trials. Int J Radiat Oncol Biol Phys 2016;96:736-747.

20. Yap ML, Sun A, Higgins J, et al. Adaptive dose escalation using serial four-dimensional positron emission tomographyl computed tomography scans during radiotherapy for locally advanced non-small cell lung cancer. Clin Oncol (R Coll Radiol) 2016;28:e199-e205.

21. Barriger RB, Fakiris AJ, Hanna N, Yu M, Mantravadi P, McGarry $\mathrm{RC}$. Dose-volume analysis of radiation pneumonitis in nonsmall-cell lung cancer patients treated with concurrent cisplatinum and etoposide with or without consolidation docetaxel. Int J Radiat Oncol Biol Phys 2010;78:1381-6.

22. Marks LB, Bentzen SM, Deasy JO, et al. Radiation dose-volume effects in the lung. Int J Radiat Oncol Biol Phys 2010;76(3 Suppl):S70-6.

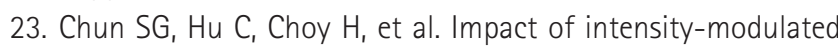
radiation therapy technique for locally advanced non-smallcell lung cancer: a secondary analysis of the NRG Oncology RTOG 0617 randomized clinical trial. J Clin Oncol 2017;35:5662.

24. Farr KP, Kallehauge JF, Moller DS, et al. Inclusion of functional information from perfusion SPECT improves predictive value of dose-volume parameters in lung toxicity outcome after radiotherapy for non-small cell lung cancer: a prospective study. Radiother Oncol 2015;117:9-16.

25. Hodge CW, Tome WA, Fain SB, Bentzen SM, Mehta MP. On the use of hyperpolarized helium MRI for conformal avoidance lung radiotherapy. Med Dosim 2010;35:297-303.

26. Vinogradskiy $Y$, Koo PJ, Castillo $R$, et al. Comparison of 4-dimensional computed tomography ventilation with nuclear medicine ventilation-perfusion imaging: a clinical validation study. Int J Radiat Oncol Biol Phys 2014;89:199-205.

27. Speirs CK, Rehman S, Molotievschi A, et al. Comprehensive analysis of dosimetric predictors of overall survival for stage III non-small cell lung cancer (NSCLC) treated with definitive radiation therapy. Int J Radiat Oncol Biol Phys 2014;90(1 Suppl);S667.

28. Tucker SL, Liu A, Gomez D, et al. Impact of heart and lung dose on early survival in patients with non-small cell lung cancer treated with chemoradiation. Radiother Oncol 2016;119:495500.

29. Chang JY, Jabbour SK, De Ruysscher D, et al. Consensus statement on proton therapy in early-stage and locally advanced non-small cell lung cancer. Int J Radiat Oncol Biol Phys 2016;95:505-16.

30. Nguyen $\mathrm{QN}$, Ly NB, Komaki R, et al. Long-term outcomes after proton therapy, with concurrent chemotherapy, for stage IIIII inoperable non-small cell lung cancer. Radiother Oncol 2015; 115:367-72.

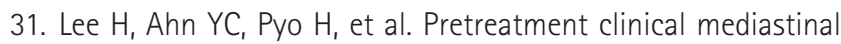
nodal bulk and extent do not influence survival in N2-positive 
stage IIIA non-small cell lung cancer patients treated with trimodality therapy. Ann Surg Oncol 2014;21:2083-90.

32. Ziel $E_{1}$ Hermann $G$, Sen $N$, et al. Survival benefit of surgery after chemoradiotherapy for stage III (NO-2) non-small-cell lung cancer is dependent on pathologic nodal response. J Thorac Oncol 2015;10:1475-80.

33. Sonett JR, Krasna MJ, Suntharalingam M, et al. Safe pulmonary resection after chemotherapy and high-dose thoracic radiation. Ann Thorac Surg 1999;68:316-20.

34. Cerfolio RJ, Bryant AS, Jones VL, Cerfolio RM. Pulmonary resection after concurrent chemotherapy and high dose (60Gy) radiation for non-small cell lung cancer is safe and may provide increased survival. Eur J Cardiothorac Surg 2009;35:718-23.

35. Cerfolio RJ, Bryant AS, Spencer SA, Bartolucci AA. Pulmonary resection after high-dose and low-dose chest irradiation. Ann Thorac Surg 2005;80:1224-30.

36. Albain KS, Swann RS, Rusch VW, et al. Radiotherapy plus chemotherapy with or without surgical resection for stage III non-small-cell lung cancer: a phase III randomised controlled trial. Lancet 2009:374:379-86.

37. Ahn S, Na K, Kim Y, et al. Surgical Salvation after full-dose concurrent chemoradiation in patients with locally advanced non-small cell lung cancer (NSCLC). Int J Radiat Oncol Biol Phys 2014;90(1 Suppl):S619.

38. Suntharalingam $M$, Paulus $R$, Edelman MJ, et al. Radiation therapy oncology group protocol 02-29: a phase II trial of neoadjuvant therapy with concurrent chemotherapy and full-dose radiation therapy followed by surgical resection and consolidative therapy for locally advanced non-small cell carcinoma of the lung. Int J Radiat Oncol Biol Phys 2012;84:456-63.

39. Senan S, Brade A, Wang LH, et al. PROCLAIM: randomized phase III trial of pemetrexed-cisplatin or etoposide-cisplatin plus thoracic radiation therapy followed by consolidation chemotherapy in locally advanced nonsquamous non-smallcell lung cancer. J Clin Oncol 2016;34:953-62.
40. Tam K, Daly M, Kelly K. Treatment of locally advanced non-small cell lung cancer. Hematol Oncol Clin North Am 2017;31:45-57.

41. Blumenschein GR Jr, Paulus R, Curran WJ, et al. Phase II study of cetuximab in combination with chemoradiation in patients with stage IIIA/B non-small-cell lung cancer: RTOG 0324. J Clin Oncol 2011;29:2312-8.

42. Kelly K, Chansky K, Gaspar LE, et al. Phase III trial of maintenance gefitinib or placebo after concurrent chemoradiotherapy and docetaxel consolidation in inoperable stage III non-small-cell lung cancer: SWOG S0023. J Clin Oncol 2008;26:2450-6.

43. Wozniak AJ, Moon J, Thomas CR Jr, et al. A pilot trial of cisplatin/etoposide/radiotherapy followed by consolidation docetaxel and the combination of bevacizumab (NSC-704865) in patients with inoperable locally advanced stage III nonsmall-cell lung cancer: SWOG S0533. Clin Lung Cancer 2015;16:340-7.

44. Saxena A, Becker D, Preeshagul I, Lee K, Katz E, Levy B. Therapeutic effects of repurposed therapies in non-small cell lung cancer: what is old is new again. Oncologist 2015;20:934-45.

45. Butts C, Socinski MA, Mitchell PL, et al. Tecemotide (L-BLP25) versus placebo after chemoradiotherapy for stage III nonsmall-cell lung cancer (START): a randomised, double-blind, phase 3 trial. Lancet Oncol 2014;15:59-68.

46. Demaria S, Formenti SC. Sensors of ionizing radiation effects on the immunological microenvironment of cancer. Int $J$ Radiat Biol 2007;83:819-25.

47. Dewan MZ, Galloway $A E_{1}$ Kawashima $N$, et al. Fractionated but not single-dose radiotherapy induces an immune-mediated abscopal effect when combined with anti-CTLA-4 antibody. Clin Cancer Res 2009;15:5379-88.

48. Seyedin SN, Schoenhals JE, Lee DA, et al. Strategies for combining immunotherapy with radiation for anticancer therapy. Immunotherapy 2015;7:967-80. 